\title{
Surgical management of thoracic myelopathy from long-segment epidural lipomatosis with skip hemilaminotomies: illustrative case
}

\author{
Matthew T. Neal, MD, Devi P. Patra, MD, and Mark K. Lyons, MD \\ Department of Neurosurgery, Mayo Clinic, Phoenix, Arizona
}

BACKGROUND Thoracic spinal epidural lipomatosis (SEL) involves the pathological overgrowth of histologically normal, unencapsulated adipose tissue that can compress the spinal cord and cause myelopathy. SEL has been associated with multiple medical conditions, including Scheuermann kyphosis (SK). Optimal treatment strategies for SEL, especially in the setting of a sagittal spinal deformity, remain unclear.

OBSERVATIONS In this report, the authors discussed surgical management of a patient with thoracic SEL and SK using skip hemilaminotomies for resection of the epidural adipose tissue. To the authors' knowledge, only one other report described a similar surgical technique in a patient who did not have a spinal deformity.

LESSONS When conservative efforts fail, thoracic SEL may require surgical treatment. Surgical planning must account for co-medical conditions such as SK. The described approach involving skip laminotomies, which minimizes spine destabilization, is a viable option to treat SEL spanning multiple spinal segments. Prognosis after surgical treatment varies and is impacted by multiple factors, including severity of preoperative neurological deficits.

https://thejns.org/doi/abs/10.3171/CASE21595

KEYWORDS thoracic myelopathy; epidural lipomatosis; Scheuermann kyphosis; spine surgery; laminectomies

Spinal epidural lipomatosis (SEL) is a rare tumor-like condition that can affect the cervical, thoracic, or lumbar spine. It involves hypertrophy and hyperplasia of rich vascularized fat tissue in the spinal canal that may result in neural compression and associated symptoms. ${ }^{1}$ The condition has been reported across broad age ranges spanning from neonates to octogenarians. ${ }^{2,3}$ The prevalence rates of SEL are relatively low, with prior radiographic studies reporting rates between $2.5 \%$ and $6.3 \%$. ${ }^{4,5}$

When present, SEL is often an incidental, radiographic finding. The radiographic appearance can be variable. In the lumbar spine, the dura on axial magnetic resonance imaging (MRI) may assume a classic trifid shape, called a " $Y$ " configuration, due to circumferential compression by the adipose tissue. ${ }^{6,7}$ In the cervical and thoracic regions, SEL has been associated with syringes. ${ }^{8,9}$

Similar to radiographic appearances, the clinical manifestations of SEL can be variable. Presenting symptoms can vary, and those symptoms may develop rapidly or insidiously. ${ }^{10,11}$ Back pain is the most common symptom associated with SEL. However, other manifestations have included radiculopathy, myelopathy, neurogenic claudication, dysesthetic pain, weakness in the limbs, and even cauda equina syndrome. $^{12}$

Reported etiologies of SEL have included exogenous steroid use, ${ }^{13}$ endogenous steroid hormonal disease, ${ }^{11,14}$ obesity, ${ }^{15,16}$ prior surgery, ${ }^{10,17}$ and idiopathic disease. ${ }^{18}$ In addition, comorbidities such as hyperlipidemia, ${ }^{19}$ acquired immunodeficiency syndrome ${ }^{20}$ Scheuermann kyphosis $(S K),{ }^{21}$ and Paget's disease of bone ${ }^{22}$ have been associated with SEL. Although multiple mechanisms likely lead to development of SEL, histological studies have shown increased inflammatory cytokines in the epidural fat, suggesting that chronic inflammation may be an underlying pathophysiological mechanism. ${ }^{23}$

Treatment for SEL is dictated by the underlying etiology. Treatment may involve weight loss, cessation of exogenous steroids, treatment of underlying endocrine abnormalities, or surgical decompression. ${ }^{24,25}$ Paradoxically, epidural steroid injections are sometimes used to treat symptomatic SEL. ${ }^{26}$ When conservative efforts fail, surgical decompression has been associated with reduction in neurological symptoms. ${ }^{8,27}$

ABBREVIATIONS MRI = magnetic resonance imaging; SEL = spinal epidural lipomatosis; SK = Scheuermann kyphosis. INCLUDE WHEN CITING Published December 13, 2021; DOI: 10.3171/CASE21595.

SUBMITTED October 17, 2021. ACCEPTED November 8, 2021.

(C) 2021 The authors, CC BY-NC-ND 4.0 (http://creativecommons.org/licenses/by-nc-nd/4.0/). 
In this report, we discuss management of a patient with thoracic SEL and SK. Although SEL is a well-documented phenomenon, many questions remain about prognosis and optimal treatment strategies, particularly in the setting of a thoracic deformity. To the author's knowledge, only one prior case report described a surgical technique similar to the one described here.

\section{Illustrative Case}

The patient is a 72-year-old man with a history of hypertension, hyperlipidemia, and type 2 diabetes mellitus who presented with a several-year history of numbness and pain in the legs. Two years earlier, he had a lumbar laminectomy at an outside institution, which did not benefit his leg numbness. He denied thoracic back pain. Over the 4 to 5 months prior to our initial meeting, his condition precipitously declined. He had progressed from independent ambulation to using a wheelchair. He had also developed worsening constipation and required self-catheterization of the bladder.

$\mathrm{He}$ had normal cranial nerve and upper extremity neurological examinations. In the right lower extremity, hip flexion was $1 / 5$, knee flexion and knee extension were $4 / 5$, ankle dorsiflexion was $1 / 5$, and pedal flexion was $3 / 5$. In the left lower extremity, hip flexion was $0 / 5$, knee flexion was $2 / 5$, knee extension was $3 / 5$, and ankle dorsiflexion and pedal flexion were $1 / 5$. He had decreased sensation distal to approximately T8 dermatome. Two beats of clonus were present in both ankles, and he had positive Babinski sign in both feet.

MRI demonstrated a nonenhancing, dorsal fatty collection measuring $1 \times 1.2 \times 12 \mathrm{~cm}$ extending from T2 to T8 vertebral levels. Occupying $>70 \%$ of the anteroposterior diameter of the spinal canal, the collection caused severe compression of the anteriorly displaced spinal cord. Hyperintense signal, consistent with myelopathy, was also noted within the cord. Tubular vascular structures were also seen within the fatty collection (Fig. 1A and B).

Because of his clinical course and imaging findings, surgical decompression was recommended. Intraoperatively, neuromonitoring modalities, including motor evoked potentials and somatosensory evoked potentials, were used. A midline incision was carried from T2 to T8. The spine was exposed in the subperiosteal plane, being careful to avoid disruption of the facet joint capsules.
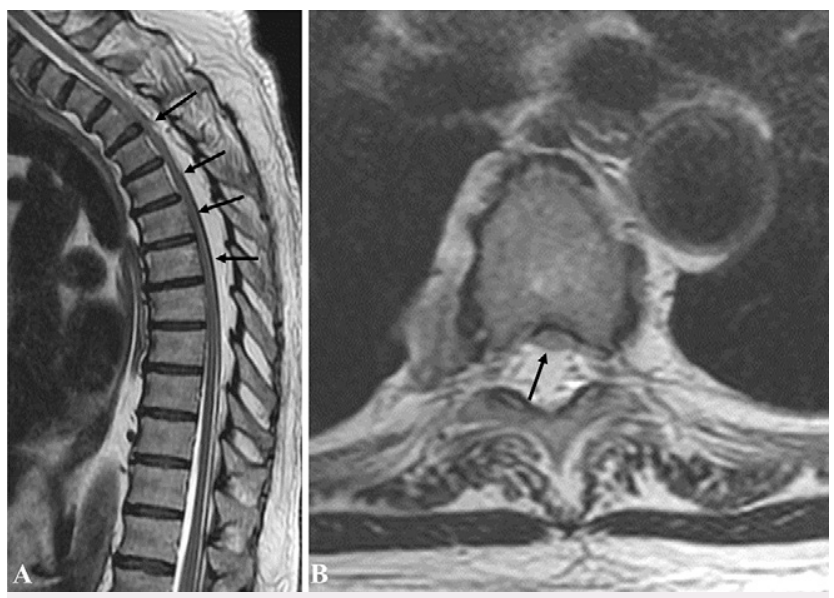

FIG. 1. A: Sagittal T2-weighted MRI demonstrating epidural lipomatosis (black arrows pointing toward the cord compression by the lipomatosis) B: Axial T2-weighted MRI demonstrating anterior displacement and compression of spinal cord due to epidural lipomatosis (black arrow).
Localization was confirmed using fluoroscopy. Hemilaminectomies along with unilateral ligamentum flavum resection were performed on the left side at T2, T4, and T6 and on the right side at T3, T5, and $\mathrm{T} 7$ using the high-speed drill and Kerrison rongeur. We preserved the spinous processes, posterior ligamentous complex, and tension band (facet capsules, interspinous ligament, supraspinous ligament, and alternating ligamentum flavum).

The adipose tissue was dissected free of the dura using spatulatype instruments, thoroughly coagulated, and removed in a piecemeal fashion. A thorough adipose tissue decompression from ipsilateral to contralateral latera recess was accomplished through the hemilaminectomy "keyholes" from T2 to T8 (Fig. 2A). Hemostasis was achieved using thrombin-based, flowable, hemostatic agents. A subfascial wound drain was left in place and removed 2 days later. Histopathological analysis demonstrated mature, adipose tissue.

After surgery, the patient completed a course of inpatient and outpatient physical therapy. Six months after surgery, the patient experienced modest improvement in his functional status. Motor strength in the lower extremities improved approximately 1 point in lower extremity muscle groups on the Oxford Scale. The patient remained dependent for transfers and mobility although less assistance was required. His Oswestry Disability Score remained in the severely disabled category, with only mild improvement $(48 \%$ to $44 \%$ ) between preoperative and 6-month postoperative time points. MRI 6 months after surgery demonstrated adequate decompression of the spinal canal and cord as well as unchanged myelomalacia
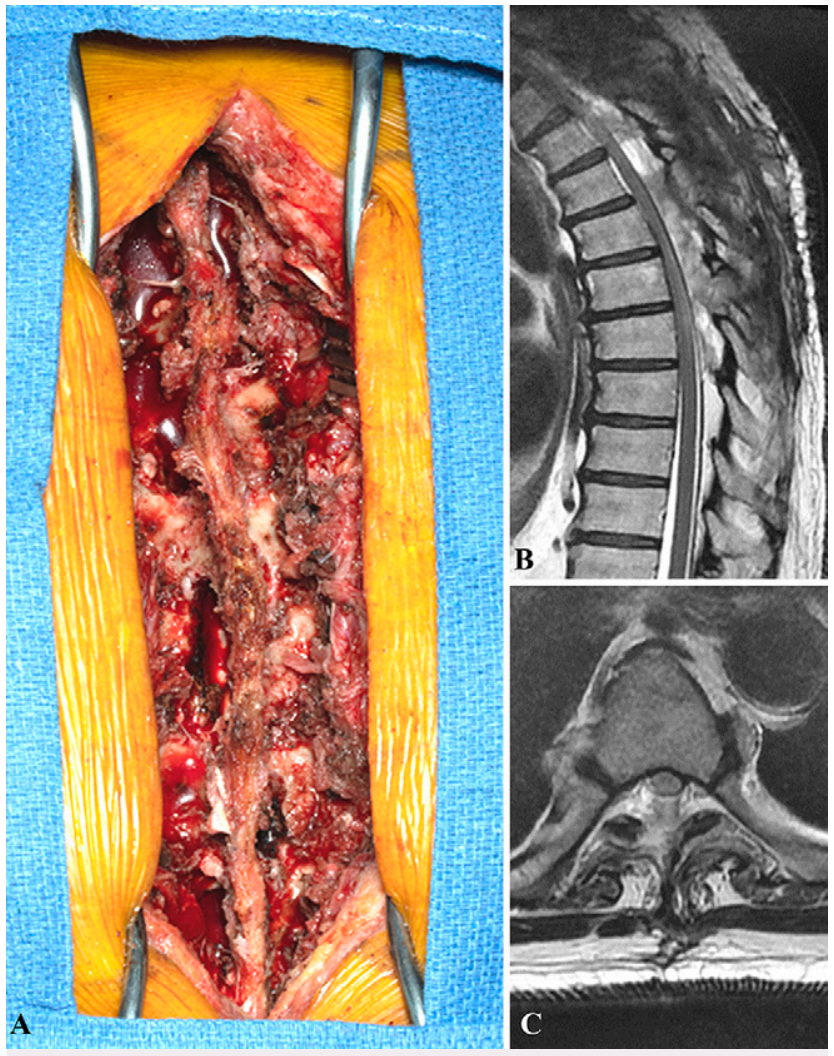

FIG. 2. A: Intraoperative photograph demonstrating skip hemilaminectomies B: 6-month postoperative sagittal T2-weighted MRI. C: 6-month postoperative axial T2-weighted MRI demonstrating decompression of spinal cord. 
(Fig. 2B and C). Upright thoracic radiographs at 6 months did not demonstrate progression of thoracic kyphosis. Longer term postoperative radiographs are not yet available.

\section{Discussion}

SEL involves the pathological overgrowth of histologically normal, unencapsulated adipose tissue, which can compress the neural elements. The patient's SEL in this case presented a unique treatment challenge because of the extent of his thoracic kyphosis in the setting of a hyperkyphotic spinal deformity.

SEL has a known association with SK. In a prior study involving 29 consecutive patients with SK, MRI demonstrated SEL in $41 \%$ of the patients. ${ }^{21}$ In another radiological study involving 86 adolescent patients with SK, $57 \%$ had SEL. ${ }^{28}$ It is not known whether there is a causal relationship or common pathophysiological mechanism between SEL and SK. Researchers have hypothesized that SEL may contribute to progressive kyphosis over time as patients continuously assume a kyphotic posture to maximize the volume of the spinal canal. ${ }^{21,29}$

Once our patient was diagnosed with SEL and SK, the next question was the treatment plan. The patient was not considered a good candidate for conservative therapies because of his rapid neurological deterioration. We attributed his myelopathy to compression from the epidural lipomatosis. Although there was draped appearance of the cord over the kyphotic segment of the thoracic spine, the patient had a more moderate case of SK and there was no evidence of spinal cord tethering on the preoperative lumbar MRI. Numerous techniques have been described to treat SEL. In both the thoracic and lumbar spine, laminoplasty, laminectomy, and fusion procedures have been described. ${ }^{3,30-32}$ Less invasive tissuepreserving decompression using endoscopic techniques has also been described. ${ }^{10,33}$

\section{Observations}

Given the patient's spinal cord compression spanning seven vertebral levels, an open surgical approach was recommended. Although the authors' preferred methods for spinal decompression involve endoscopic and tubular retractors, the time and additional patient risk associated with the extensive minimally invasive spine (MIS) decompression did not outweigh the benefits. In addition, hemostasis through MIS portals can be challenging because of robust venous bleeding and extensive dead space after spinal canal decompression. Meticulous hemostasis is critical to avoid postoperative epidural hematoma, a reported complication from treating SEL. ${ }^{34}$ We did not recommend spinal fusion because the patient did not have a functional limitation or back pain associated with his rigid, kyphotic deformity. Avoidance of spinal fusion was also desired because the patient had an extensive smoking history and poor bone quality, which placed him at higher risk for instrumentation loosening and failed fusion. We also wanted to avoid full laminectomies in an effort to preserve as much of his posterior tension band as possible and prevent development of back pain and progressive kyphosis. We therefore proposed alternating skip hemilaminectomies to accomplish the needed decompression while minimizing spinal destabilization. A single case report has described a similar technique for thoracic SEL in which alternating hemilaminotomies are used for decompression. The patient in the prior report did not have a spinal deformity. The authors highlighted the same advantages of avoiding instrumentation and maximizing postoperative spinal stability. ${ }^{35}$

Reported outcomes after surgical treatment of SEL have ranged from moderate ${ }^{9,32,34,36}$ to excellent. ${ }^{3,10,18,35-41}$ The reason for this variability is likely multifactorial. In this case, we presume that several factors contributed to the more modest neurological improvement. The patient had cord compression over a long segment, and SEL involving multiple vertebral levels has been associated with greater preoperative deficit. ${ }^{42}$ The patient had thoracic SEL, as opposed to lumbar SEL, which is associated with lower rates of complete neurological recovery. ${ }^{43}$ The patient had a prolonged duration of symptoms ( $>2$ years). During this time, his neurological deficits progressed significantly. The severity of presenting symptoms, particularly ambulatory status, has portended a poor prognosis in prior studies. ${ }^{43}$

\section{Lessons}

When conservative efforts fail, thoracic SEL may require surgical treatment. Surgical planning must account for co-medical conditions such as SK. The described approach involving skip laminotomies, which minimizes spine destabilization, is a viable option for treating SEL that spans multiple spinal segments. Prognosis after surgical treatment is variable and is impacted by multiple factors, including severity of preoperative neurological deficits. Delay in diagnosis and surgical treatment of SEL due to decreased awareness or knowledge about the entity may lead to worse clinical outcomes.

\section{References}

1. Artner J, Leucht F, Cakir B, Reichel H, Lattig F. Spinal epidural lipomatosis. Article in German. Orthopade. 2012;41(11):889-893.

2. Singh J, Sodhi M, Arora N. Neonatal epidural lipomatosis: a rare case. J Pediatr Neurosci. 2018;13(2):267-269.

3. Okunlola Al, Orewole TO, Okunlola CK, Babalola OF, Akinmade A. Epidural lipomatosis in elderly patient: a rare cause of cauda equina compression. Surg Neurol Int. 2021;12:7.

4. Theyskens NC, Paulino Pereira NR, Janssen SJ, Bono CM, Schwab JH, Cha TD. The prevalence of spinal epidural lipomatosis on magnetic resonance imaging. Spine J. 2017;17(7):969-976.

5. Malone JB, Bevan PJ, Lewis TJ, Nelson AD, Blaty DE, Kahan ME. Incidence of spinal epidural lipomatosis in patients with spinal stenosis. J Orthop. 2017;15(1):36-39.

6. Spinnato P, Lotrecchiano L, Ponti F. "Y" sign in spinal epidural lipomatosis. Joint Bone Spine. 2021;88(1):105056.

7. Kuhn MJ, Youssef HT, Swan TL, Swenson LC. Lumbar epidural lipomatosis: the "Y" sign of thecal sac compression. Comput Med Imaging Graph. 1994;18(5):367-372.

8. Sáez-Alegre M, Pérez López C, Giner García J, et al. Epidural lipomatosis and syringomyelia in adulthood: case report and literature review. World Neurosurg. 2019;129:341-344.

9. Arora M, Leever JD. Thoracic epidural lipomatosis associated with syrinx: a case report. Radiol Case Rep. 2020;15(5):528-530.

10. Youn MS, Woo YH, Shin JK. Rapid progression of spinal epidural lipomatosis after percutaneous endoscopic spine surgery mimicking disc herniation. Int J Surg Case Rep. 2020;73:1-4.

11. Lotan I, Charlson RW, Fatterpekar GM, et al. Progressive myelopathy associated with spinal epidural lipomatosis in three non-obese patients with type 1 diabetes mellitus. J Neurol Sci. 2020;411: 116688.

12. Kim SS, Lim DJ. Epidural lipomatosis with cauda equina syndrome in chronic alcoholic patient: a case report. Int J Surg Case Rep. 2017;33:12-15. 
13. Fessler RG, Johnson DL, Brown FD, Erickson RK, Reid SA, Kranzler L. Epidural lipomatosis in steroid-treated patients. Spine (Phila $\mathrm{Pa}$ 1976). 1992;17(2):183-188.

14. Ahmad S, Best T, Lansdown A, et al. Spinal epidural lipomatosis: a rare association of Cushing's disease. Endocrinol Diabetes Metab Case Rep. 2020;2020.

15. Spinnato P, Ponti F, de Pasqua S. MRI Diagnosis of obesity-related spinal epidural lipomatosis. Can J Neurol Sci. 2020;47(1):124-125.

16. Zhang B, Yuan H, Hu L, Saad M. Obesity is a risk factor for epidural lipomatosis: a meta-analysis. J Orthop Surg (Hong Kong). 2021;29(2):23094990211027391.

17. Greenish D, Watura K, Harding I. Spinal epidural lipomatosis following bilateral spinal decompression surgery. BMJ Case Rep. 2019;12(2):e226985.

18. Kellett CG, Siva V, Norman ICF, Jung J, Grahovac G, Minhas P. Symptomatic idiopathic spinal epidural lipomatosis in 9 patients: clinical, radiologic, and pathogenetic features. World Neurosurg. 2019;126:e33-e40.

19. Ishihara S, Fujita N, Yagi M, et al. Idiopathic spinal epidural fat accumulation is associated with hyperlipidemia. Spine (Phila Pa 1976). 2018:43(8):E468-E473.

20. Schürmann D, Rademaker J, Trottenberg T, Bergmann F, Wesselmann H, Suttorp N. Spinal epidural lipomatosis: a manifestation of HAART-associated lipodystrophy. AIDS. 2005;19(17): 2052-2054.

21. Abul-Kasim K, Schlenzka D, Selariu E, Ohlin A. Spinal epidural lipomatosis: a common imaging feature in Scheuermann disease. J Spinal Disord Tech. 2012;25(7):356-361.

22. Koziarz P, Avruch L. Spinal epidural lipomatosis associated with Paget's disease of bone. Neuroradiology. 2002;44(10):858-860.

23. Fujita N, Hosogane N, Hikata T, et al. Potential involvement of obesity-associated chronic inflammation in the pathogenesis of idiopathic spinal epidural lipomatosis. Spine (Phila Pa 1976). 2016; 41(23):E1402-E1407.

24. Trungu S, Forcato S, Raco A. Spinal epidural lipomatosis: weight loss cure. World Neurosurg. 2019;125:368-370.

25. Valcarenghi J, Bath O, Boghal H, Ruelle M, Lambert J. Benefits of bariatric surgery on spinal epidural lipomatosis: case report and literature review. Eur J Orthop Surg Traumatol. 2018;28(7): $1437-1440$.

26. McCormick Z, Plastaras C. Transforaminal epidural steroid injection in the treatment of lumbosacral radicular pain caused by epidural lipomatosis: a case series and review. J Back Musculoskeletal Rehabil. 2014;27(2):181-190.

27. Kim K, Mendelis J, Cho W. Spinal epidural lipomatosis: a review of pathogenesis, characteristics, clinical presentation, and management. Global Spine J. 2019;9(6):658-665.

28. Lonner BS, Toombs CS, Mechlin M, et al. MRI screening in operative Scheuermann kyphosis: is it necessary? Spine Deform. 2017;5(2):124-133.

29. Zhang Z, Liu Z, Zhu Z, Qiu Y. Spinal epidural lipomatosis: an easily ignored secondary intraspinal disorder in spinal kyphotic deformities. BMC Musculoskelet Disord. 2017:18(1):112.

30. Yang K, Ji C, Luo D, Li K, Xu H. Lumbar laminotomy and replantation for the treatment of lumbar spinal epidural lipomatosis: a case report. Medicine (Baltimore). 2021;100(30):e26795.

31. Tardivo V, Scudieri C, Bruzzo M, Lupidi F. Acute neurologic decline in a patient with spinal stenosis: blame it on the epidural fat. $\mathrm{Br} \mathrm{J}$ Neurosurg. Published online July 28, 2021. doi: 10.1080/ 02688697.2021 .1958149$.

32. Ulrich NH, Gravestock I, Winklhofer S, et al. Two-year outcome comparison of decompression in 14 lipomatosis cases with 169 degenerative lumbar spinal stenosis cases: a Swiss prospective multicenter cohort study. Eur Spine J. 2020;29(9):2243-2253.

33. Frank E. Endoscopic suction decompression of idiopathic epidural lipomatosis. Surg Neurol. 1998;50(4):333-335.

34. Ferlic PW, Mannion AF, Jeszenszky D, et al. Patient-reported outcome of surgical treatment for lumbar spinal epidural lipomatosis. Spine J. 2016;16(11):1333-1341.

35. Martínez Santos JL, Saway B, Damon AC, Varma A. Multilevel hemilaminotomy windows for the surgical management of spinal epidural lipomatosis causing syringomyelia: technical note and literature review. World Neurosurg. 2021;148:4-12.

36. Yasuda T, Suzuki K, Kawaguchi Y, et al. Clinical and imaging characteristics in patients undergoing surgery for lumbar epidural lipomatosis. BMC Musculoskelet Disord. 2018;19(1):66.

37. Han X, Xu D, Ren Z, Chen X, Li Z, Li S. Lumbar spinal stenosis combined with obesity-induced idiopathic spinal epidural lipomatosis treated with posterior lumbar fusion: case report. BMC Surg. 2021;21(1):215.

38. Gérardy F, Dewandre Q, Lombard A, et al. Epidural lipomatosis : management proposal. Article in French. Rev Med Liege. 2021;76(2):71-76.

39. Chaudhary K, Chandy D, Agrawal V, Dhawale A, Nagaonkar S. Spinal epidural lipomatosis with osteoporotic vertebral fractures presenting as acute thoracic myelopathy in a 33-year-old man with Cushing disease. World Neurosurg. 2020;142:136-141.

40. Kang SS, Lee SC, Kim SK. A novel percutaneous biportal endoscopic technique for symptomatic spinal epidural lipomatosis: technical note and case presentations. World Neurosurg. 2019; 129:49-54.

41. Bayerl SH, Dinkelbach M, Heiden P, Prinz V, Finger T, Vajkoczy P. Treatment results for lumbar epidural lipomatosis: does fat matter? Eur Spine J. 2019;28(1):69-77.

42. Ishikawa $Y$, Shimada $Y$, Miyakoshi N, et al. Decompression of idiopathic lumbar epidural lipomatosis: diagnostic magnetic resonance imaging evaluation and review of the literature. J Neurosurg Spine. 2006;4(1):24-30.

43. Praver M, Kennedy BC, Ellis JA, D'Amico R, Mandigo CE. Severity of presentation is associated with time to recovery in spinal epidural lipomatosis. J Clin Neurosci. 2015;22(8):1244-1249.

\section{Disclosures}

Dr. Neal reported personal fees from Medtronic Inc. outside the submitted work

\section{Author Contributions}

Conception and design: Neal. Analysis and interpretation of data: Lyons. Drafting the article: all authors. Critically revising the article: all authors. Reviewed submitted version of manuscript: all authors. Approved the final version of the manuscript on behalf of all authors: Neal. Administrative/technical/material support: Neal. Study supervision: Neal.

\section{Correspondence}

Matthew T. Neal: Mayo Clinic College of Medicine and Science, Phoenix, AZ. neal.matthew@mayo.edu. 